\title{
Stochastic Stability of Adaptive Quantizers for Markov Sources
}

\author{
Serdar Yüksel \\ Mathematics and Engineering and Department of Mathematics and Statistics, \\ Queen's University, Kingston, Ontario, Canada, K7L 3N6 \\ Email: yuksel@mast.queensu.ca
}

\begin{abstract}
A stochastic stability result for a class of adaptive quantizers which were introduced by Goodman and Gersho is presented. We consider a case where the input process is a linear Markov source which is not necessarily stable. We present a stochastic stability result for the estimation error and the quantizer, thus generalizing the stability result of Goodman and Gersho to a Markovian, and furthermore to an unstable, setting. Furthermore, it is shown that, there exists a unique invariant distribution for the state and the quantizer parameters under mild irreducibility conditions. The second moment under the invariant distribution is finite, if the system noise is Gaussian.
\end{abstract}

\section{INTRODUCTION}

A quantizer, $Q$, for a scalar continuous variable is a map $\mathbb{R} \rightarrow \mathbb{R}$, characterized by a sequence of bins $\left\{\mathcal{B}_{i}\right\}$ and their representation $\left\{q^{i}\right\}$, such that $\forall i, Q(x)=q^{i}$ if and only if $x \in \mathcal{B}_{i}$.

Of particular interest is the class of uniform quantizers. In the following, we modify the description of a traditional uniform quantizer by assigning the same value when the state is in the overflow region of the quantizer. As such, a uniform quantizer: $Q: \mathbb{R} \rightarrow \mathbb{R}$ with step size $\Delta$ and an (odd) $K$ number of levels satisfies the following for $k=1,2 \ldots, K$ :

$$
Q_{K}^{\Delta}(x)=\left(\frac{-(K+1)}{2}+k\right) \Delta, \quad \text { if } \quad x \in\left[\gamma_{1}, \gamma_{2}\right)
$$

with $\left[\gamma_{1}, \gamma_{2}\right)=\left[\left((k-1)-\frac{K}{2}\right) \Delta,\left(k-\frac{-K}{2}\right) \Delta\right)$. If $|x|>\frac{K}{2} \Delta$ or $x=\frac{K}{2} \Delta$, we let the quantizer output be null, denoting that the state is outside the granular region of the quantizer. The value will be taken to be zero at the receiver.

A general class of quantizers are those which are adaptive. Let $\mathbb{S}$ be a set of states for a quantizer state $S$. Let $F: \mathbb{S} \times \mathbb{R} \rightarrow$ $\mathbb{S}$ be a state update-function. An adaptive quantizer has the following state update equations:

$$
S_{t+1}=F\left(S_{t}, Q_{t}\left(x_{t}\right)\right)
$$

Here, $Q_{t}$ is the quantizer applied at time $t, x_{t}$ is the input to the quantizer $Q_{t}$, and $S_{t}$ is the state of the quantizer. We note that, such a quantizer is implementable since the updates can be performed both at the encoder and the decoder.

One particular class of adaptive quantizers is introduced by Goodman and Gersho [2]. One such type has the following form with $Q_{K}^{\Delta}$ being a uniform quantizer with $K$ bins and binsize $\Delta$ and $\bar{Q}$ determining the updates in the bin-size of the uniform quantizer as a function of the source and the current bin size:

$$
\begin{aligned}
q_{t} & =Q_{K}^{\Delta_{t}}\left(x_{t}\right) \\
\Delta_{t+1} & =\Delta_{t} \bar{Q}\left(x_{t}, \Delta_{t}\right)
\end{aligned}
$$

Here $\Delta_{t}$ characterizes the uniform quantizer, as it is the bin size of the quantizer at time $t$. In the model that we will consider, $\bar{Q}($.$) will take ratio between x$ and $\Delta$ as its input.

We consider an LTI discrete-time scalar system described by

$$
x_{t+1}=a x_{t}+d_{t},
$$

where $x_{t}$ is the state at time $t$, and $\left\{d_{t}\right\}$ is a sequence of zero-mean independent, identically distributed (i.i.d.) random variables which satisfy the following:

Assumption A: The $\left\{d_{t}\right\}$ sequence is such that each of the random variables admits a probability distribution $\nu$ which is absolutely continuous with respect to the Lebesgue measure on $\mathbb{R}$, and for every open set $D \in \mathbb{R}, \nu(D)>0$. Furthermore, $E\left[d_{t}^{2}\right]=E\left[d^{2}\right]<\infty$.

Later we will consider a stronger assumption:

Assumption B: The sequence $\left\{d_{t}\right\}$ is an i.i.d. Gaussian sequence with zero-mean and a finite variance.

Remark: With Assumption B, we will obtain stronger results in the development of the paper. We could generalize Assumption $B$, by requiring the tail of the noise distributions to decay exponentially fast.

In the above, $a$ is the system coefficient with $|a| \geq 1$, that is, the system is open-loop unstable. The case with $|a|<1$ (stable case), can be considered as a special case of our setting, as will be apparent in the development of the paper.

In the following, we discuss the quantization policy and the notion of stability that we use in the main result. Before, however, we present a brief review of relevant literature.

\section{A. Literature Review}

There is large literature on adaptive quantizer design. One important reference here is the work by Goodman and Gersho [2], where an adaptive quantizer was introduced and the adaptive quantizer's stationarity properties were investigated when the source fed to the quantizer is independent and identically distributed with a finite second moment. Kieffer and Dunham [3] have obtained conditions for the stochastic stability of a 
number of coding schemes when the source considered is also stable, that is when it has an invariant distribution; where various forms of stability of the quantizer and the estimation error have been studied. In our case, however, the schemes in [2] and [3] are not directly applicable, as the process we consider is open-loop unstable, as well as Markovian.

Zooming type adaptive quantizers, which will be described further in the paper, have been recently introduced by Brockett and Liberzon [4] for the use in remote stabilization of openloop unstable, noise-free systems with arbitrary initial conditions. Nair and Evans [1] provided a stability result under the assumption that the quantization is variable-rate and showed the first result for a noisy setup (with unbounded support for the noise probability measure) that on average it suffices to use more than $\log _{2}(|a|)$ bits to achieve a form of stability. [1] used asymptotic quantization theory to obtain a time-varying scheme, where the quantizer is used at certain intervals at a very high rate, and at other times, the quantizer is not used. We provide an approach which allows us to both provide a result for the case when the quantizer is fixed-rate as well as to obtain an invariance condition for a probability measure on the quantizer parameters.

\section{Main Results of the Paper}

We first present a brief overview of stochastic stability for Markov Chains. The reader is referred to [6] for further discussions.

\section{A. Stochastic Stability of Markov Chains}

Let $\left\{x_{t}, t \geq 0\right\}$ be a Markov chain with state space $(\mathbb{X}, \mathcal{B}(\mathbb{X}))$, and defined on a probability space $(\Omega, \mathcal{F}, \mathcal{P})$, where $\mathcal{B}(\mathbb{X})$ denotes the Borel $\sigma$-field on $\mathbb{X}, \Omega$ is the sample space, $\mathcal{F}$ a sigma field of subsets of $\Omega$, and $\mathcal{P}$ a probability measure. Let $P(x, D):=P\left(x_{t+1} \in D \mid x_{t}=x\right)$ denote the transition probability from $x$ to $D$, that is the probability of the event $\left\{x_{t+1} \in D\right\}$ given that $x_{t}=x$ for $D \in \mathcal{B}(\mathbb{X})$.

Definition 2.1: For a Markov chain with transition probability defined as before, a probability measure $\pi$ is invariant on the Borel space $(\mathbb{X}, \mathcal{B}(\mathbb{X}))$ if

$$
\pi(D)=\int_{\mathbb{X}} P(x, D) \pi(d x), \quad \forall D \in \mathcal{B}(\mathbb{X}) .
$$

Definition 2.2: A Markov chain is $\mu$-irreducible, if for any set $B \subset \mathbb{X}$, such that $\mu(B)>0$, and $\forall x \in \mathbb{R}$, there exists some integer $n>0$, possibly depending on $B$ and $x$, such that $P^{n}(x, B)>0$, where $P^{n}(x, B)$ is the transition probability in $n$ stages, that is $P\left(x_{t+n} \in B \mid x_{t}=x\right)$.

Definition 2.3: A set $A \subset \mathbb{X}$ is $\mu$-petite on $(\mathbb{X}, \mathcal{B}(\mathbb{X}))$ if for some distribution $\mathcal{T}$ on $\mathbb{N}$ (set of natural numbers), and some non-trivial measure $\mu$,

$$
\sum_{n=0}^{\infty} P^{n}(x, B) \mathcal{T}(n) \geq \mu(B), \quad \forall x \in A, \quad B \in \mathcal{B}(\mathbb{X}) .
$$

Definition 2.4: Consider a Markov process $\left\{x_{t}\right\}$ taking values in $\mathbb{X}$. A compact set $A \in \mathbb{X}$, is recurrent if $P(\min (t>$ $\left.\left.0: x_{t} \in A\right)<\infty \mid x_{0}=x\right)=1, \forall x \in A$.
Theorem 2.1: ([6] Theorem 4.1) Let $A \in \mathbb{X}$ be a $\mu$-petite set which is recurrent. If the Markov Chain is $\mu$-irreducible, and if $\sup _{x \in A} E\left[\min \left(t>0: x_{t} \in A\right) \mid x_{0}=x\right]<\infty$, then the Markov chain is positive Harris recurrent and it admits a unique invariant distribution.

The existence of an invariant distribution is important primarily because of the following Theorem:

Theorem 2.2 (Birkhoff's Individual Ergodic Theorem): Consider a positive Harris recurrent Markov process $\left\{x_{t}\right\}$ taking values in $\mathbb{X}$, with invariant distribution $\pi($.$) . Let$ $f: \mathbb{X} \rightarrow \mathbb{R}$ be a bounded function, measurable on $\mathcal{B}(\mathbb{X})$. Then, the following holds almost surely:

$$
\lim _{T \rightarrow \infty} \frac{1}{T} \sum_{t=0}^{T-1} f\left(x_{t}\right)=\int f(x) \pi(d x) .
$$

\section{B. Main Results}

Before proceeding further with the presentation of the main results, we further discuss the adaptive quantizers considered. An example of Goodman-Gersho [2] type adaptive quantizers are those that have zoom level coefficients as the quantizer state. In the zooming scheme, the quantizer enlarges the bin sizes in the quantizer until the state process is in the range of the quantizer, where the quantizer is in the perfect-zoom phase. Due to the effect of the system noise, occasionally the state will be in the overflow region of the quantizer, leading to an under-zoom phase. We will refer to such quantizers as zooming quantizers.

In the following, we will assume the communication channel to be a discrete noiseless one with capacity $R$.

Theorem 2.3: Consider a zooming type adaptive quantizer applied to the linear system described by (2), under Assumption A. If the noiseless channel has capacity, for some $\epsilon>0$,

$$
R=\log _{2}(\lceil|a|+\epsilon\rceil+1),
$$

then, there exists an adaptive quantization policy such that there exists a compact set $S$ with

$$
\sup _{x \in S} E\left[\min \left(t>0: x_{t} \in S\right) \mid x_{0}=x\right]<\infty,
$$

thus $S$ is a recurrent set.

The proof of this Theorem is given in Section II-C. In particular, with $R=\log _{2}(\lceil|a|+\epsilon\rceil+1), K=2^{R}$, let us define $R^{\prime}=\log _{2}\left(2^{R}-1\right)$. We will now consider the following update rules. For $t \geq 0$ and with $\Delta_{0} \in \mathbb{R}$ selected arbitrarily and $\hat{x}_{-1}=0$, consider the following:

$$
\begin{aligned}
\hat{x}_{t} & =a \hat{x}_{t-1}+Q_{K}^{\Delta_{t}}\left(z_{t}\right) \\
z_{t} & =a x_{t-1}-a \hat{x}_{t-1}+d_{t-1} \\
\Delta_{t+1} & =\Delta_{t} \bar{Q}\left(\left|\frac{z_{t}}{\Delta_{t} 2^{R^{\prime}-1}}\right|\right)
\end{aligned}
$$

If there exist $\delta, \epsilon, \eta>0$ with $\eta<\epsilon$ and $L>\frac{|a|+\epsilon-\eta}{|a|}$ such that,

$$
\begin{array}{cll}
\bar{Q}(x) \geq|a|+\delta & \text { if } & |x|>1 \\
\frac{|a|}{|a|+\epsilon-\eta}<\bar{Q}(x)<1 & \text { if } & 0 \leq|x| \leq 1, \Delta>L \\
\bar{Q}(x)=1 & \text { if } & 0 \leq|x| \leq 1, \Delta \leq L(4)
\end{array}
$$


with

$$
\frac{\sqrt{E\left[d_{t}^{2}\right]}}{L \frac{|a|}{|a|+\epsilon-\eta} 2^{R^{\prime}-1}}<\delta,
$$

we will show that a recurrent set exists with the update rules described above.

Our result on the existence and uniqueness of an invariant distribution is the following.

Theorem 2.4: Under the setup of Theorem 2.3, for a zooming quantizer, if the quantizer bin sizes are such that their (base-2) logarithms are integer multiples of some scalar $s$ and $\log _{2}(\bar{Q}()$.$) take values in integer multiples of s$, and the integers taken are relatively prime (that is they share no common divisors except for 1), then the jointly Markov process $\left(x_{t}-\hat{x}_{t}, \Delta_{t}\right)$ forms a positive (Harris) recurrent Markov chain, and, as such, has a unique invariant distribution.

The proof of this result is presented in Section II-D.

The findings above can be used to present a stronger result (with the additional Assumption B), the proof of which is omitted:

Theorem 2.5: Under the setups of Theorem 2.3, Theorem 2.4 and Assumption B, it follows that

$$
\lim _{t \rightarrow \infty} E\left[\left(x_{t}-\hat{x}_{t}\right)^{2}\right]<\infty
$$

and this limit is independent of the initial states of the quantizer and the system.

Remark: We note that the stability result for such a scheme requires new techniques to be used, as classical tools in Markovian stability theory will not be applicable directly. This is mostly because of the fact that, the quantizer helps reduce the uncertainty on the system state only when the state is in the granular region of the quantizer. The times when the state is in this region are random. As such, in the following section, we use a two-stage Martingale approach to prove the existence of a recurrent set, and subsequently the stability results.

\section{Proof of Theorem 2.3: Recurrence}

Consider the update equations (3)-(4). Toward the proof, we will first obtain a supporting result. Let us define, for $t \in \mathbb{Z}_{+}$,

$$
e_{t}:=x_{t}-\hat{x}_{t} .
$$

Lemma 2.1: Let $\mathcal{B}\left(\mathbb{R} \times \mathbb{R}_{+}\right)$denote the Borel $\sigma$-field on $\mathbb{R} \times \mathbb{R}_{+}$. It follows that $\forall(C \times D) \in \mathcal{B}\left(\mathbb{R} \times \mathbb{R}_{+}\right)$,

$$
\begin{aligned}
& P\left(\left(e_{t}, \Delta_{t}\right) \in(C \times D) \mid\left(e_{t-1}, \Delta_{t-1}\right), \ldots,\left(e_{0}, \Delta_{0}\right)\right) \\
& =P\left(\left(e_{t}, \Delta_{t}\right) \in(C \times D) \mid\left(e_{t-1}, \Delta_{t-1}\right)\right),
\end{aligned}
$$

i.e. $\left(e_{t}, \Delta_{t}\right)$ is a Markov chain.

Let us define $h_{t}:=\frac{z_{t}}{\Delta_{t} 2^{R^{\prime}-1}}$. Consider the following sets:

$$
C_{e}=\left\{z:|z| \leq E^{\prime}\right\} \quad C_{h}=\{h:|h| \leq 1\},
$$

with $E^{\prime}=2^{R^{\prime}-1} E$, with $E=L\left(\frac{|a|}{|a|+\epsilon-\eta}\right)$. Further, let another set be $C_{e}^{\prime}=\left\{z:|z| \leq F^{\prime}\right\}$, and $F^{\prime}=2^{R^{\prime}-1} F$ with a sufficiently large $F$ value to be derived below. We will study the expected number of time stages between visits of $\left\{\left(e_{t}, h_{t}\right)\right\}$ to $C_{e}^{\prime} \times C_{h}$. Consider the drift of the $\left(e_{t}, h_{t}\right)$ process in Figure 1: When $\left(e_{t}, h_{t}\right)$ are in $C_{e} \times C_{h}$, the expected drift increases both $|h|$ and $|e|$. When the $\left\{z_{t}\right\}$ process gets outside $C_{e}^{\prime}$ and $\left\{h_{t}\right\}$ outside $C_{h}$ (under-zoomed), there is a drift for $\left\{h_{t}\right\}$ towards $C_{h}$, however, $\left|e_{t}\right|$ will keep increasing on average. Finally, when the process hits $C_{h}$ (perfect-zoom), then the process drifts towards $C_{e}^{\prime}$. There exists an upper bound on the value that $h$ can take when $\left\{e_{t}\right\}$ is inside the compact sets.

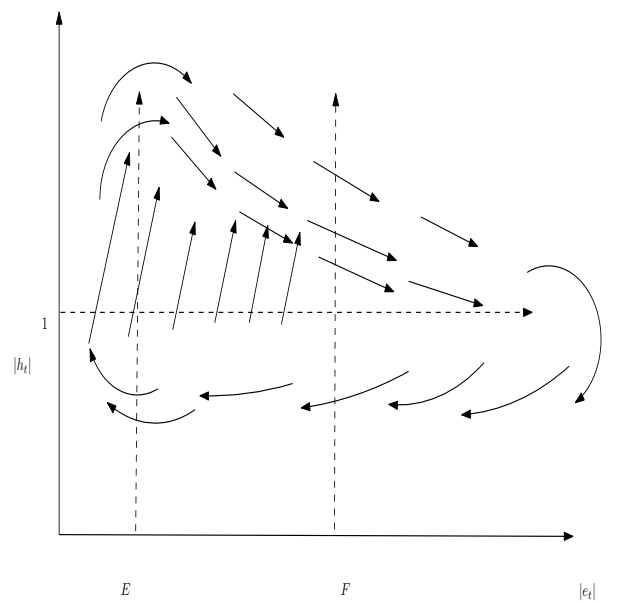

Fig. 1: Drift in the Error Process: When under-zoomed, the error increases on average; when perfectly-zoomed, the error decreases.

We first show that the sequence $\left\{h_{t}, t \geq 0\right\}$ visits $C_{h}$ infinitely often with probability 1 and the expected length of the excursion is uniformly bounded over all possible values of $(z, h) \in C_{e}^{\prime} \times C_{h}$. Once $C_{h}$ is visited, then the estimation error decreases on average. However, unless this is met, $\left|z_{t}\right|$ and $\left|e_{t}\right|$ keeps increasing stochastically. Let $V\left(h_{t}\right)=h_{t}^{2}$ serve as a Lyapunov function. Define a sequence of stopping times for the perfect-zoom case with

$$
\begin{aligned}
\tau_{0} & =0, \\
\tau_{z+1} & =\inf \left\{k>\tau_{z}:\left|h_{k}\right| \leq 1\right\}, \quad z \in \mathbb{Z}_{+}
\end{aligned}
$$

We have that, if $\left|h_{t}\right|>1$ (under-zoomed)

$$
E\left[h_{t+1}^{2} \mid \Delta_{t}, h_{t}\right] \leq \frac{\left(a^{2}+\frac{E\left[d^{2}\right]}{\left|z_{t}\right|^{2}}\right)}{(a+\delta)^{2}}\left(h_{t}\right)^{2}
$$

Since when $\left|h_{t}\right|>1$, we have that $\left|z_{t}\right|>2^{R^{\prime}-1} L\left(\frac{|a|}{|a|+\epsilon-\eta}\right)$, it follows that

$$
E\left[h_{t+1}^{2} \mid \Delta_{t}, h_{t}\right] \leq\left(\frac{a^{2}+\frac{E\left[d^{2}\right]}{E^{\prime 2}}}{(a+\delta)^{2}}\right)\left(h_{t}\right)^{2} .
$$

If $\left|h_{t}\right| \leq 1$, then

$$
\begin{aligned}
E\left[h_{t+1}^{2}\right] & \leq \frac{a^{2} \frac{\left(\Delta_{t}\right)^{2}}{4}+E\left[d_{t}^{2}\right]}{\left(\Delta_{t} 2^{R^{\prime}-1}\right)^{2}}\left(\frac{|a|+\epsilon-\eta}{|a|}\right)^{2} \\
& \leq \frac{a^{2} \frac{L^{\prime 2}}{4}+E\left[d_{t}^{2}\right]}{\left(L^{\prime} 2^{R^{\prime}-1}\right)^{2}}\left(\frac{|a|+\epsilon-\eta}{|a|}\right)^{2} \\
& =: K_{1},
\end{aligned}
$$


where $L^{\prime}=L \frac{|a|}{|a|+\epsilon-\eta}$ (this is a lower bound on $\Delta_{t}$ ). Hence, with it follows that

$$
E\left[h_{t+1}^{2}-h_{t}^{2} \mid \Delta_{t}, h_{t}\right] \leq-\rho h_{t}^{2}+K_{1} 1_{\left(\left|h_{t}\right| \leq 1\right)},
$$

where $1_{(U)}$ is the indicator function for event $U$ with

$$
\begin{aligned}
K_{1} & =\frac{a^{2} \frac{L^{\prime 2}}{4}+E\left[d_{t}^{2}\right]}{\left(L^{\prime} 2^{R^{\prime}-1}\right)^{2}}\left(\frac{|a|+\epsilon-\eta}{|a|}\right)^{2}, \\
\rho & =1-\frac{\left(a^{2}+\frac{E\left[d^{2}\right]}{E^{\prime 2}}\right)}{(a+\delta)^{2}}
\end{aligned}
$$

Since for $A, B>0, A^{2}+B^{2} \leq(A+B)^{2}$ it follows that the hypothesis $\sqrt{\frac{E\left[d_{t}^{2}\right]}{L^{\prime} 2^{R^{\prime}}-1}}<\delta$ in the theorem statement ensures $\rho>0$. Now, define $M_{0}:=V\left(h_{0}\right)$, and for $t \geq 1$

$$
M_{t}:=V\left(h_{t}\right)-\sum_{i=0}^{t-1}\left(-\rho+K_{1} 1_{\left(h_{i} \in C_{h}\right)}\right)
$$

Define a stopping time:

$$
\tau^{N}=\min \left(N, \min \left\{i>0: V\left(h_{i}\right) \geq N, V\left(h_{i}\right) \leq 1\right\}\right) .
$$

As $\left|h_{t}\right|>1$ when $h_{t} \notin C_{h}, E\left[M_{t+1} \mid\left(e_{s}, h_{s}\right), s \leq t\right] \leq$ $M_{t}, \quad \forall t \geq 0$, it follows that, $\left\{M_{t}\right\}$ is a Super-Martingale sequence. The stopping time $\tau^{N}$ is bounded and the SuperMartingale sequence is also bounded for $t \leq \tau^{N}$. Hence, we have, by the Martingale optional sampling theorem: $E\left[M_{\left(\tau^{N}\right)}\right] \leq E\left[M_{0}\right]$. Hence, we obtain

$$
E\left[\sum_{i=0}^{\tau^{N}-1}\right] \rho \leq V\left(h_{0}\right)+K_{1} E\left[\sum_{i=0}^{\tau^{N}-1} 1_{\left(h_{i} \in C\right)}\right]
$$

Thus, $\rho E\left[\tau^{N}-1+1\right] \leq V\left(h_{0}\right)+K_{1}$, and by the Monotone Convergence Theorem, $\rho \lim _{N \rightarrow \infty} E\left[\tau^{N}\right]=\rho E[\tau] \leq$ $V\left(h_{0}\right)+K_{1}=1+K_{1}$. Hence,

$$
E\left[\tau_{z+1}-\tau_{z}\right] \leq\left(1+K_{1}\right) / \rho
$$

uniformly for all $h_{\tau_{z}} \in C_{h}$. Once perfect-zooming occurs, that is $h_{t} \in C_{h}$, then we have $E\left[e_{t+1}^{2} \mid \Delta_{t}, h_{t}\right] \leq \frac{a^{2}}{2^{2 R^{\prime}}} \frac{\Delta_{t}^{2}}{4}+$ $E\left[d_{t}^{2}\right]$. By the strong Markov property, $\left(\Delta_{\tau_{z}}, h_{\tau_{z}}\right)$ is also a Markov chain as $\left\{\tau_{z}<n\right\} \in \mathcal{F}_{n}$, the filtration generated by the quantizer state and the quantizer output at time $n$, for any $n \geq \tau_{z}$. The probability that $\tau_{z+1} \neq \tau_{z}+1$, is upper bounded by the expression

$P_{e}\left(\Delta_{\tau_{z}}\right):=P\left(d_{\tau_{z}}^{2}>\left(\Delta_{\tau_{z}}(|a| / 2)\left(\frac{\lceil|a|+\epsilon\rceil-|a|-\epsilon+\eta}{|a|+\epsilon-\eta}\right)\right)^{2}\right)$ If $\tau_{z+1} \neq \tau_{z}+1$, then this means that the error is increasing on average and the system is once-again under-zoomed at time $t=\tau_{z}+1: e_{\tau_{z}+1}=a e_{\tau_{z}}+d_{\tau_{z}}$ with $\Delta_{\tau_{z}+1}=\frac{|a|}{|a|+\epsilon-\eta} \Delta_{\tau_{z}}$. With some probability, the quantizer will still be in the perfectzoom phase: $\tau_{z+1}=\tau_{z}+1$. In case perfect-zoom is lost, there is a uniform bound on when the zoom is expected to be recovered.

We can, after some omitted steps, show that, there exist $\psi>0,|G|<\infty$ such that

$$
E\left[\log \left(\Delta_{\tau_{z+1}}^{2}\right) \mid \Delta_{\tau_{z}}, h_{\tau_{z}}\right] \leq \log \left(\Delta_{\tau_{z}}^{2}\right)-\psi+G 1_{\left(\left|\Delta_{\tau_{z}}\right| \leq F^{\prime}\right)},
$$

$$
F^{\prime}=\frac{\sqrt{E\left[d^{2}\right]}}{K_{2} \sqrt{\frac{-\psi-2 \log \left(\frac{|a|}{|a|+\epsilon-\eta}\right)}{\left\{\left(2\left(1+K_{1}\right) / \rho\right) \log (|a|+\delta)\right\}}}},
$$

and $G=2 \log (F)+2\left(\left(1+K_{1}\right) / \rho\right) \log (|a|+\delta)+\psi$.

Hence, we have obtained another drift condition for the sampled Markov chain. This shows that the newly constructed process $\Delta_{\tau_{z}}$ hits $C_{e}^{\prime}=\left\{\Delta:|\Delta| \leq F^{\prime}\right\}$ infinitely often. Let us call this stopping time process $\tau_{y}^{\prime}$ and define it by $\tau_{0}^{\prime}=\tau_{0}=0$, for $y>0$,

$$
\begin{aligned}
\tau_{y+1}^{\prime} & =\tau_{y}^{\prime}+\inf \left\{t>\tau_{y}^{\prime}:\left|\Delta_{t}\right| \leq F^{\prime},\left|h_{t}\right| \leq 1 \mid\right\} \\
& =\tau_{y}+k
\end{aligned}
$$

with $k=\inf \left\{l>0:\left|\Delta_{\tau_{z+l}}\right| \leq F^{\prime},\left|h_{\tau_{z+l}}\right| \leq 1, \tau_{y}^{\prime}=\tau_{z}\right\}$. Hence, $k$ is the number of visits such that $\left\{h:\left|h_{t}\right| \leq 1\right\}$ until $e_{t}$ hits $C_{e}^{\prime}$. When there is an excursion outside this set, the expected length of the trip (in terms of the new Markov process) is finite, that is $E\left[\tau_{1}^{\prime}\right]<\infty$. This follows because of the following: Define, $M_{0}=\log \left(\Delta_{0}^{2}\right)$, and for some sufficiently small $\alpha>0$,

$$
M_{\tau_{z}}=\log \left(\Delta_{\tau_{z}}^{2}\right)-\left(\sum_{l=0}^{\tau_{z}-1}-\alpha+G 1_{\left(\left|\Delta_{\tau_{z}}\right| \leq F^{\prime}\right)}\right) .
$$

$M_{\tau_{z}}$ is a Super-Martingale sequence for $0 \leq \tau_{z} \leq \tau_{1}^{\prime}$ if $\alpha<$ $\frac{\psi^{z}}{\frac{1+K_{1}}{\rho}}$, since

$$
E\left[M_{\tau_{z+1}} \mid \mathcal{F}_{\tau_{z}}\right] \leq M_{\tau_{z}}
$$

For any finite $n$, let us define $k^{n}=(\min (k, \min (l>$ $\left.\left.0: l+\log \left(\Delta_{\tau_{l}}^{2}\right) \geq n\right)\right)$, which is a stopping time. Hence, $E\left[\sum_{l=0}^{k^{n}-1} \alpha\right] \leq M_{0}+G$. Since $\psi \geq \alpha E\left[\tau_{l+1}-\tau_{l}\right]$, it follows that $\alpha E\left[\sum_{l=1}^{k^{n}} \tau_{l}-\tau_{l-1}\right] \leq M_{0}+G$, or $E\left[k^{n}\right] \leq \frac{M_{0}+G}{\alpha}$. Finally, taking the limit as $n \rightarrow \infty$, and by the Monotone Convergence Theorem, it follows that $E\left[\tau_{1}^{\prime}\right] \leq \frac{M_{0}+G}{\alpha}$ and $\sup _{\left(\Delta_{\tau_{z}}, h_{\tau_{z}}\right) \in C_{e}^{\prime} \times C_{h}} E\left[\tau_{1}^{\prime}\right] \leq \frac{M_{0}+G}{\alpha}$.

D. Proof of Theorem 2.4: Existence and Uniqueness of an Invariant Probability Distribution

In our setting, $\left(e_{t}, \Delta_{t}\right)$ form the Markov chain, as was proved in Lemma 2.1. Before proceeding further, let us recall the following, which follows from an important result in number theory, known as Bézout's Lemma.

Lemma 2.2: ([2] Theorem 2) Let $\{-A, B\}$ be two integers such that $A>0, B>0$. Let $\mathbb{I}$ be the set of all integers that can be obtained by summing positive integer multiples of elements in $\{-A, B\}$. If $A, B$ are relatively prime, then $\mathbb{I}=\mathbb{Z}$, that is, $\mathbb{I}$ is the set of all integers.

In view of the above results, we now show that the set of bin sizes forms a communication class under the hypothesis of the theorem: Since we have $\Delta_{t+1}=\bar{Q}\left(\left|\frac{z_{t}}{\Delta_{t} 2^{R^{\prime}-1}}\right|\right) \Delta_{t}$, it follows that

$$
\log _{2}\left(\Delta_{t+1}\right) / s=\log _{2}\left(\bar{Q}\left(\left|\frac{z_{t}}{\Delta_{t} 2^{R^{\prime}-1}}\right|\right)\right) / s+\log _{2}\left(\Delta_{t}\right) / s,
$$


is also an integer. Furthermore, since the source process $x_{t}$ is Lebesgue-irreducible (as the system noise admits a continuous probability density function with positive mass on every open set), and there is a uniform lower bound $L^{\prime}$ on bin-sizes, the error process takes values in any of the admissible quantizer bins with non-zero probability.

Let the values taken by $\log _{2}\left(\bar{Q}\left(\left|\frac{z_{t}}{\Delta_{t^{\prime}-1}}\right|\right)\right) / s$ be $\{-A, B\}$. By the hypothesis of the theorem statement, $A, B$ are relatively prime. Consider two integers $k, l \geq \frac{\log _{2}\left(L^{\prime}\right)}{s}$. Further, assume, without any loss of generality that $l>k$. From $k$ to $l$, one can construct a sequence consisting of $-A$ and $B$ integers such that the sum of these integers equals $l-k$ for all $l, k \in \mathbb{N}$, that is there exist $N_{A}, N_{B} \in \mathbb{Z}_{+}$such that

$$
l-k=-N_{A} A+N_{B} B .
$$

Consider first the case where $k>\frac{\log _{2}\left(L^{\prime}\right)}{s}+N_{A} A$. We show that the probability of $N_{A}$ occurrences of perfect zoom, and $N_{B}$ occurrences of under-zoom phases is bounded away from zero. This set of occurrences includes the event that in the first $N_{A}$ time stages perfect-zoom occurs and later, successively, $N_{B}$ times under-zoom phase occurs. The probability of this event is lower bounded by

$$
\left.\left(P\left(d_{t} \in\left[-|a| 2^{s k}-L^{\prime},-|a| 2^{s k}+L^{\prime}\right]\right)\right)^{N_{A}}\left(P\left(d_{t}>|a| 2^{s l}\right]\right)\right)^{N_{B}}
$$

which is positive. A similar analysis can be performed when $k<\frac{\log _{2}\left(L^{\prime}\right)}{s}+N_{A} A$, by considering the opposite order of events, where in the first $N_{B}$ times, under-zoom occurs, and in the successive $N_{A}$ time stages, perfect-zoom occurs. As such, the selection of these events will always have non-zero probability due to the Lebesgue irreducibility of the noise distribution. Hence, for any two integers $k, l$ and for some $p>0, P\left(\log _{2}\left(\Delta_{t+p}\right)=l s \mid \log _{2}\left(\Delta_{t}\right)=k s\right)>0$.

In our setting, the recurrent set consists of both $\Delta_{t}$ and $e_{t}$. Hence, in this setting, the product space is the product of a discrete-space and a real space. For $e_{t}$, the Borel sets are the open intervals, which are visited infinitely often, and for $\Delta_{t}$, the individual atoms are visited infinitely often with probability 1 .

Now, we can connect the results of the previous section with Theorems 2.3 and 2.1. The recurrent set $C_{e}^{\prime} \times C_{h}$ is $\nu$-petite, for some probability measure $\nu$ as any Borel set in the state space is visited starting from $C_{e}^{\prime} \times C_{h}$, and the chain is irreducible. These two imply that the chain is positive Harris recurrent.

\section{Case with a Stable Source}

The stable source also follows from the analysis in the paper, since the state process, and the bin size process visit a compact set infinitely often, under the conditions presented. We note that, when $a=0$, the setting of Goodman and Gersho is obtained.

\section{Simulation}

As a simulation study, we consider a linear system with the following dynamics: $x_{t+1}=2.5 x_{t}+d_{t}$, where $E\left[d_{t}\right]=$ $0, E\left[d_{t}^{2}\right]=1$, and $\left\{d_{t}\right\}$ are i.i.d. Gaussian variables. We use the zooming quantizer with rate $\log _{2}(4)=2$, since 4 is the smallest integer as large as $\lceil 2.5\rceil+1$. We have taken $L^{\prime}=1$. Figure 2 corroborates the stochastic stability result, by explicitly showing the under-zoomed and perfectly zoomed phases, with the peaks in the plots showing the under-zoom phases.
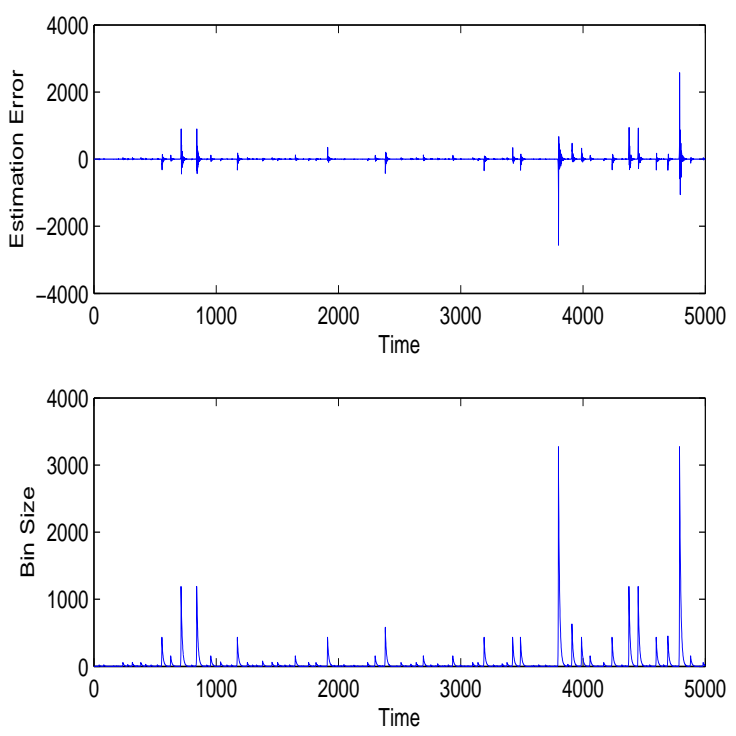

Fig. 2: Time horizon is 5000 .

\section{REFERENCES}

[1] G. N. Nair and R. J. Evans, "Stabilizability of stochastic linear systems with finite feedback data rates", SIAM Journal on Control and Optimization, vol. 43, pp. 413 - 436, July 2004.

[2] D. J. Goodman and A. Gersho, "Theory of an adaptive quantizer", IEEE Trans. Commun., vol. 22, pp. 1037- 1045, Aug. 1974.

[3] J. C. Kieffer and J. G. Dunham "On a type of stochastic stability for a class of encoding schemes", IEEE Trans. Inform. Theory, vol. 29, pp. 793-797, November 1983.

[4] R. Brockett and D. Liberzon, "Quantized feedback stabilization of linear systems",IEEE Trans. on Automatic Control, vol. 45, pp. 1279-1289, July 2000.

[5] W. S. Wong and R. W. Brockett, "Systems with finite communication bandwidth constraints - part II: Stabilization with limited information feedback, IEEE Trans. Automatic Control, 42(9):1294-1299, September 1997.

[6] S. P. Meyn and R. Tweedie, "Stability of Markovian processes I: Criteria for discrete-time chains", Adv. Appl. Probability, vol. 24, pp. 542-574, 1992. 